\title{
Protocolo de Tratamento de Cefaleia na Emergência em um Hospital-Escola
}

\author{
Protocol of Treatment of Headache in the Emergency in a University hospital
}

Eliana Meire Melhado, Izabela Dias Brugugnolli, Guiherme Vedovato Vilela de Salis, Carolina Buck, Lilian Audi Goulart, Talita Alvarez Sucena, Juliana Vilaça Vaz, Pedro Matheus Benelli, Maria Emilia Miani Pereira, Renata Perri Soares Ferreira

Faculdades Integradas Padre Albino - FIPA - Catanduva, SP, Brasil

Melhado EM, Brugugnolli ID, Salis GW, Buck C, Goulart LA, Sucena TA, et al. Protocolo de Tratamento de Cefaleia na Emergência em um Hospital-Escola. Headache Medicine. 2017;8(2):43-7

\section{RESUMO}

Objetivo: Nas Unidades de Urgência e Emergência (UUE), cefaleias são responsáveis por $16 \%$ das queixas principais. Destes casos, $90 \%$ são cefaleias de causas primárias. Por isso o objetivo do estudo foi implantar um protocolo de atendimento às cefaleias na UUE de um HospitalEscola. Método: A busca na literatura científica foi o método utilizado, pesquisando-se bibliograficamente, os sites da Bireme, MedLine, Scielo, Lilacs e PubMed. Após a revisão do tema, montou-se um protocolo que foi implantado no pronto-atendimento de um Hospital-Escola. Resultado: $O$ manejo adequado da cefaleia na UUE depende primeiramente do diagnóstico adequado, segundo os critérios da Sociedade Internacional de Cefaleia (ICHD-3beta) 2013, o qual deve excluir causas de cefaleia secundária, por exemplo, traumas, infecções, massas intracranianas, hemorragias. Quanto ao tratamento farmacológico, deve-se seguir o protocolo abaixo. Administrar dipirona $\mathrm{lg}$ IV (intravenoso). Se evoluir bem o paciente é liberado e orienta-se acompanhamento ambulatorial. Caso contrário, opta-se por Clorpromazina 0,5-1 mg/kg IV diluída em SF 0,9\% $500 \mathrm{ml}$ por 2 horas, ou Clorpromazina $0,1 \mathrm{mg} / \mathrm{kg}$ em bolo IV. Caso paciente apresente vômito, considerar uso de Ondansetrona $8 \mathrm{mg}$ em SF 0,9\% 100ml em 15min, ou Dimenidrinato IV. Caso resposta desfavorável usar Cetoprofeno 100mg IV em SF 0,9\% $100 \mathrm{ml}$ em 30min com protetor gástrico. Caso persista, utilizar Dexametasona 10mg IV e se necessário repetir $4 \mathrm{mg}$ IV. Se não houver melhora da dor após essas medidas, internar paciente para investigação. Quando a resposta for boa libera-se o paciente. Deve-se então desencorajar o abuso crônico de analgésicos e orientar o paciente a procurar tratamento especializado. A utilização de opioides no tratamento da crise migranosa na sala de emergência deve ser desencorajada, tanto pela falta de comprovação de eficácia como pelo risco de induzir à cronificação da migrânea. Conclusões: A abordagem da cefaleia primaria na UUE deve-se basear em protocolos padrões que sistematizem o atendimento ao paciente de maneira a minimizar os custos e efetivar o tratamento.

Palavras-chave: Emergência; Cefaleia; Tratamento

\section{INTRODUÇÃO}

Dentre os sintomas mais comuns na prática clínica, a cefaleia se constitui um dos principais, visto que, na maioria das vezes, pode se relacionar a patologias graves. Mesmo as cefaleias consideradas benignas são prejudiciais para os indivíduos que as possuem, pois em grande parcela tornam-se o agente incapacitante para realização de suas funções cotidianas. Em pequena escala, a cefaleia acarreta problemas pessoais como a própria abstenção no trabalho, nas relações sociais e atividades. Já em grande escala, trata-se de um problema de saúde pública com impacto socioeconômico. ${ }^{(1,2)}$

Segundo o Subcomitê de Classificação das Cefaleias da Sociedade Internacional de Cefaleia, ${ }^{(3)}$ há mais de duzentos tipos de cefaleias descritas. É fundamental que o médico possa primeiramente dividir as cefaleias entre primárias, quando não está presente nenhuma outra doença subjacente e secundárias, quando a dor existe como 
sintoma de outra condição. A cefaleia primária possui como subtipos a migrânea, cefaleia do tipo tensional, cefaleias em salvas ou trigêmino-autonômicas e outros tipos de cefaleias primárias, enquanto as secundárias englobam transtornos atribuídos a um determinado fator. ${ }^{(3,4)}$

Estima-se que a prevalência da cefaleia ao longo da vida na população seja de superior a $90 \%$. As cefaleias primárias são causas comuns de procura por auxílio médico, estando responsável por aproximadamente $7 \%$ dos atendimentos não agendados em unidades básica de saúde e $1 \%$ a $3 \%$ dos atendimentos em unidades de emergência.

Dada a grande importância desse sintoma e sua elevada prevalência a anamnese é indispensável para se chegar ao diagnóstico, devendo ser baseada no protocolo de atendimento de cefaleias. Isso é comprovado pela ausência de exames complementares capazes de realizar o diagnóstico por si. ${ }^{(5)}$

O diário de acompanhamento da dor de cabeça é uma ferramenta útil para a detecção de fatores desencadeantes, bem como a verificação de melhora com o tratamento estabelecido. Porém este acompanhamento é ambulatorial, portanto fora da emergência. ${ }^{(6)}$
O objetivo do estudo foi implantar um protocolo de atendimento às cefaleias na UUE para os médicos que atendem em serviços de emergência.

\section{MÉTODO}

A busca na literatura científica foi o método utilizado, pesquisando-se bibliograficamente, os sites da Bireme, MedLine, Scielo, Lilacs e PubMed. Após a revisão do tema, montou-se um protocolo que foi implantado no prontoatendimento de um hospital-escola.

\section{RESULTADOS}

O manejo adequado da cefaleia na UUE deve seguir um algoritmo para o tratamento (Diagrama 1), e depende primeiramente do diagnóstico adequado, segundo os critérios da Sociedade Internacional de Cefaleia (ICHD-3-beta) 20137, o qual deve excluir causas de cefaleia secundária, por exemplo, traumas, infecções, massas intracranianas, hemorragias. Os sinais de alerta e de benignidade da cefaleia, e os exames que devem ser solicitados, 8 são descritos respectivamente nas Tabelas 1, 2 e 3



Diagrama 1. Algoritmo do tratamento da Cefaleia na UEE 
Tabela 1 - Sinais de alerta para uma cefaleia na $U_{U E}^{9}$

Primeiro episódio de cefaleia de início súbito

A pior cefaleia da vida;

Cefaleia de início súbito (atinge a intensidade máxima em até 1 minuto);

Evolução subaguda (dias a semanas) com intensidade e/ou frequência crescentes;

Mudança de padrão ou surgimento de nova cefaleia superposta a antiga ou em paciente enxaquecoso;

Cefaleia iniciada em paciente com idade superior a 50 anos;

Cefaleia em pacientes com sintomas sistêmicos (febre, perda de peso, descarga nasal purulenta, elevação súbita da pressão arterial, espessamento das artérias temporais superficiais);

Cefaleia em paciente com história de HIV ou câncer;

Cefaleia em paciente com glaucoma ou outras afecções oculares agudas;

Mudança de padrão da aura e aura persistente e prolongada;

Mudança de padrão da aura e aura persistente e prolongada;

Cefaleia em pacientes com história de convulsões ou TCE recente;

Anormalidade no exame neurológico (incluindo alterações da consciência ou linguagem, da motricidade ocular, do campo visual e das funções motoras e sensitivas, além de papiledema e sinais de irritação meníngea; sintomas/sinais neurológicos irritativos ou deficitários ou déficit neurológico persistente);

Lateralidade fixa ou localização exclusivamente occipital;

Cefaleia desencadeada por tosse, exercício físico, atividade sexual ou manobra de Valsalva;

Acompanhada por distúrbio de consciência, febre, rigidez de nuca;

Alterações na frequência, na intensidade, ou nas características clínicas da crise de cefaleia;

Cefaleia progressiva ou nova diária persistente;

Sintomas neurológicos que não preenchem os critérios para migrânea com aura típica;

Alteração na pele ou órbita sugestivas de mal formação arteriovenosa;

Comorbidade de crises epilépticas parciais;

Acompanhada de distúrbios endócrinos ou hipertensão arterial sistêmica

Acorda o paciente durante o sono;

Ausência de melhora com tratamento adequado;

Acorda o paciente à noite.

Tabela 2 - Sinais de benignidade da cefaleia

Cefaleias anteriores idênticas

Sem outros sintomas (exceto náuseas, vômitos, fotofobia e fonofobia)

Nivel de consciência preservado e comportamento adequado

Sem qualquer dúvida quanto aos sinais meníngeos

Exame clínico normal incluindo sinais vitais

Tabela 3 - Exames mais apropriados para investigação

Tomografia computadorizada de crânio e/ou seios da face de acordo com a indicação de cada caso;

Punção lombar com exame completo do líquido cefalorraquidiano de acordo com a indicação de cada caso;

Exames laboratoriais e/ou radiológicos na suspeita de doenças sistêmicas:

Os abaixo não serão realizados na emergência;

Ressonância magnética de crânio incluindo sequências adequadas a cada caso; RM - angioRM-VenoRM
Quanto ao tratamento farmacológico, deve-se seguir o protocolo adiante..$^{(10)}$

Administrar dipirona $1 \mathrm{~g} \mathrm{IV} \mathrm{(intravenoso).} \mathrm{Se} \mathrm{evoluir} \mathrm{bem}$ o paciente é liberado e orienta-se acompanhamento ambulatorial. Caso contrário, opta-se por clorpromazina 0,5-1 mg/kg IV diluída em SF 0,9\% $500 \mathrm{ml}$ por duas horas, ou clorpromazina $0,1 \mathrm{mg} / \mathrm{kg}$ em bolo IV. Caso paciente apresente vômito, considerar uso de ondansetrona $8 \mathrm{mg}$ em SF 0,9\% $100 \mathrm{ml} \mathrm{em} 15 \mathrm{~min}$, ou dimenidrinato IV. Caso resposta desfavorável usar cetoprofeno 100 mg IV em SF 0,9\% $100 \mathrm{ml} \mathrm{em} 30$ min com protetor gástrico. Caso persista, utilizar dexametasona 10 mg IV e se necessário repetir 4 mg IV. Se não houver melhora da dor após essas medidas, internar paciente para investigação. Quando a resposta for boa libera-se o paciente. Deve-se então desencorajar o abuso crônico de analgésicos e orientar o paciente a procurar tratamento especializado (Diagrama 2). 


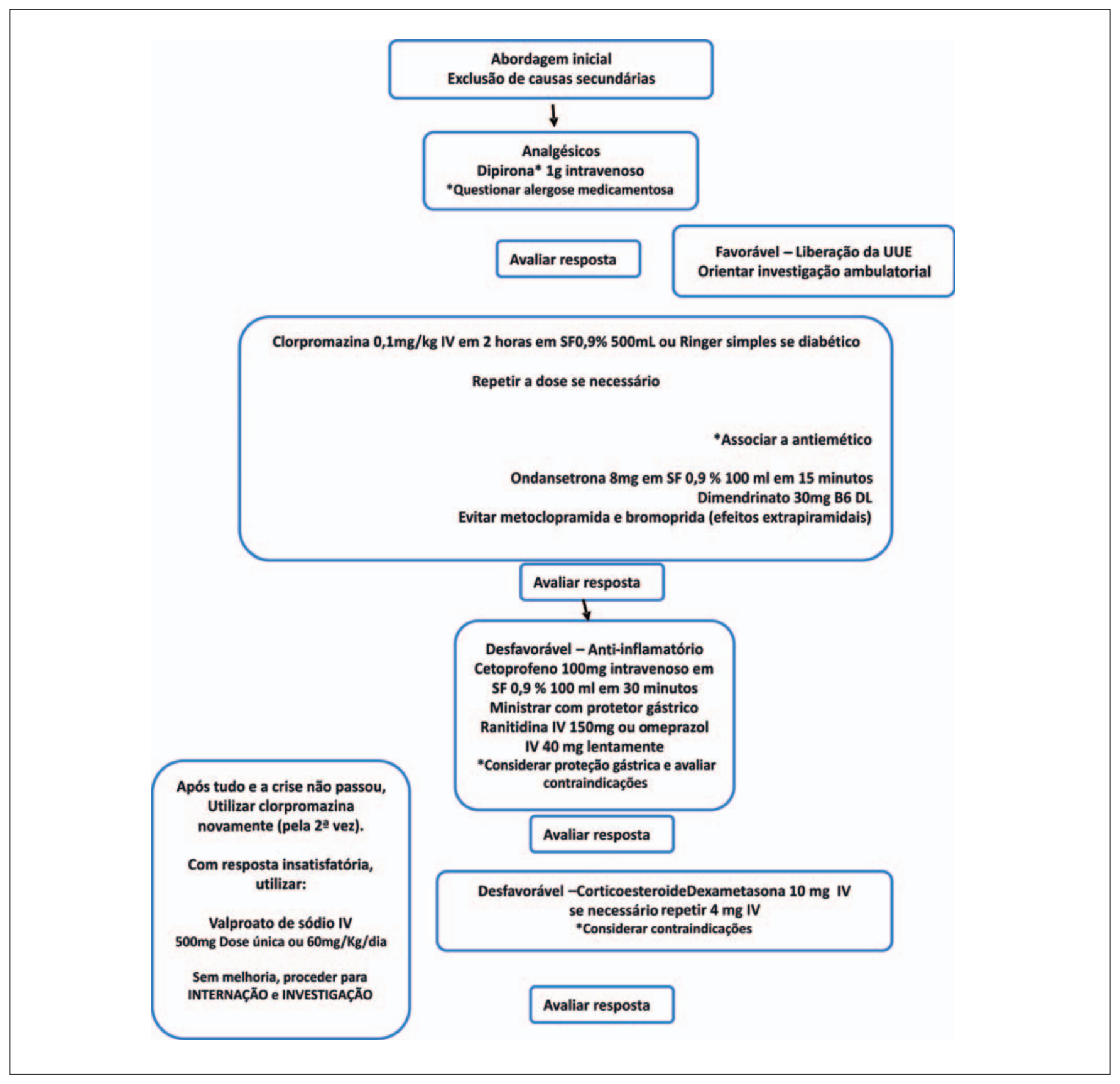

Diagrama 2. Protocolo de tratamento farmacológico

A utilização de opioides no tratamento da crise migranosa na sala de emergência deve ser desencorajado, tanto pela falta de comprovação de eficácia como pelo risco de induzir à cronificação da migrânea.

\section{CARACTERIZAÇÃO DA RESPOSTA FAVORÁVEL}

- Alívio total ou significativo (permite retorno às atividades rotineiras) da dor;
- Ausência ou diminuição suficiente de náuseas, vômitos, desconforto e/ou mal estar, bem como quaisquer outros sintomas associados à migrânea;

- Reassegurar que o (a) paciente e seus familiares estão cientes e esclarecidos quanto ao diagnóstico e seguros quanto a conduta adequada;

- Passar para o paciente um diário de dor de cabeça para anotar as dores em frequência e intensidade até o paciente consultar com especialista; 
- Não iniciar tratamento preventivo ao paciente;

- Encaminhar para consulta médica ambulatorial ( Via Unidade de Saúde da Família). ${ }^{(8,10)}$

Instruções pós-alta

- Encaminhar para consulta médica ambulatorial (Unidade de Saúde da Família)

- Orientar o retorno a emergência em caso de:

- Piora ou mudança do padrão da cefaleia

- Febre

- Vômitos ou incapacidade de ingerir líquidos

- Tonteira, vertigem, desequilíbrio ou dificuldade de marcha

- Alterações visuais, motoras ou de linguagem

- Outras anormalidades que o perturbem. ${ }^{(8,10)}$

\section{CONCLUSÃO}

A abordagem da cefaleia primária na UUE deve se basear em protocolos padrões que sistematizem o atendimento ao paciente de maneira a minimizar os custos e efetivar o tratamento.

\section{REFERÊNCIAS}

1. Speciali JG. Classificação das cefaléias. Medicina, Ribeirão Preto, 30:421-7, out./dez. 1997.

2. Raskin NH. Headache. In: Harrison TR (ed). Principles of Internal Medicine. Edl3. New York: McGraw-Hill, 1994: 65-71.

3. The International Classification of Headache Disorders. 3nd Edition (beta version). Cephalalgia. 2013;33(9):629-808

4. Porto CC. Semiologia médica, $2^{a}$ ed. Rio de Janeiro, Guanabara Koogan, 2 , 1994.

5. Dhopesh V, Anwar R, Herring C. A retrospective assessment of emergency department patients with complaint of headache. Headache 1979;9:37-42.

6. Melhado EM. Diário de Cefaleia. In Cefaleia na Mulher. Sanvito WL, ed. Atheneu, São Paulo, pp245-246. 2011

7. Headache Classification Subcommittee of the International Headache Society (IHS). The International Classification of Headache Disorders 3nd Edition (beta version). Cephalalgia, 33(9), p. 629-808, 2013.

8. Bordini CA, Roesler C, Carvalho DS, Macedo DD, Piovesan É; Melhado EM, et al. Recommendations for the treatment of migraine attacks - a Brazilian consensus. Arq Neuropsiquiatr; ; 74(3): 262-271, Mar. 2016

9. Contag SA, Mertz HL, Bushnell CD. Migraine during pregnancy: is it more than a headache? Nat Rev Neurol. 2009; 5:449-56.

10. Lilian Audi Goulart, Talita Alvarez Sucena, Juliana Vilaça Vaz, Pedro Matheus Benelli, Natalia Gonçalves Rodrigues, Izabela Dias Brugugnolli, Eliana Meire Melhado. Cefaleia na emergência/Headache in emergency. Ciência, Pesquisa e Consciência. 2014 janeiro/dezembro; 6(1):33-37.
11. Cerbo R, Villani V, Bruti G, Di Stani F, Mostardini C. Primary headache in Emergency Department: prevalence, clinical features and therapeutical approach. J Headache Pain. 2005 Sep;6(4):287-9.

12. Friedman BW, Grosberg BM. Diagnosis and management of the primary headache disorders in the emergency department setting. Emerg Med Clin North Am. 2009 February;27(1): 71 viii.

13. Consenso da Sociedade Brasileira de Cefaleia. Recomendações para o tratamento da crise migranosa. Arq Neuropsiquiatr. 2000;58:371-89.

$$
\begin{array}{r}
\text { Correspondência } \\
\text { Eliana Meire Melhado, } \\
\text { Faculdades Integradas Padre Albino - FIPA } \\
\text { Rua dos Estudantes, } 225 \\
15.809 \text { - } 144 \text { - Catanduva, SP, Brasil } \\
\text { Tel.: (17) 331 1-3328 }
\end{array}
$$

Recebido: 06 de novembro de 2016

Aceito: 05 de fevereiro de 2017 